Document downloaded from:

http://hdl.handle.net/10251/143135

This paper must be cited as:

Ribes, A.; Santiago Felipe, S.; Aviñó, A.; Candela-Noguera, V.; Eritja, R.; Sancenón Galarza, F.; Martínez-Máñez, R.... (12-2). Design of oligonucleotide-capped mesoporous silica nanoparticles for the detection of miRNA-145 by duplex and triplex formation. Sensors and Actuators B Chemical. 277:598-603. https://doi.org/10.1016/j.snb.2018.09.026

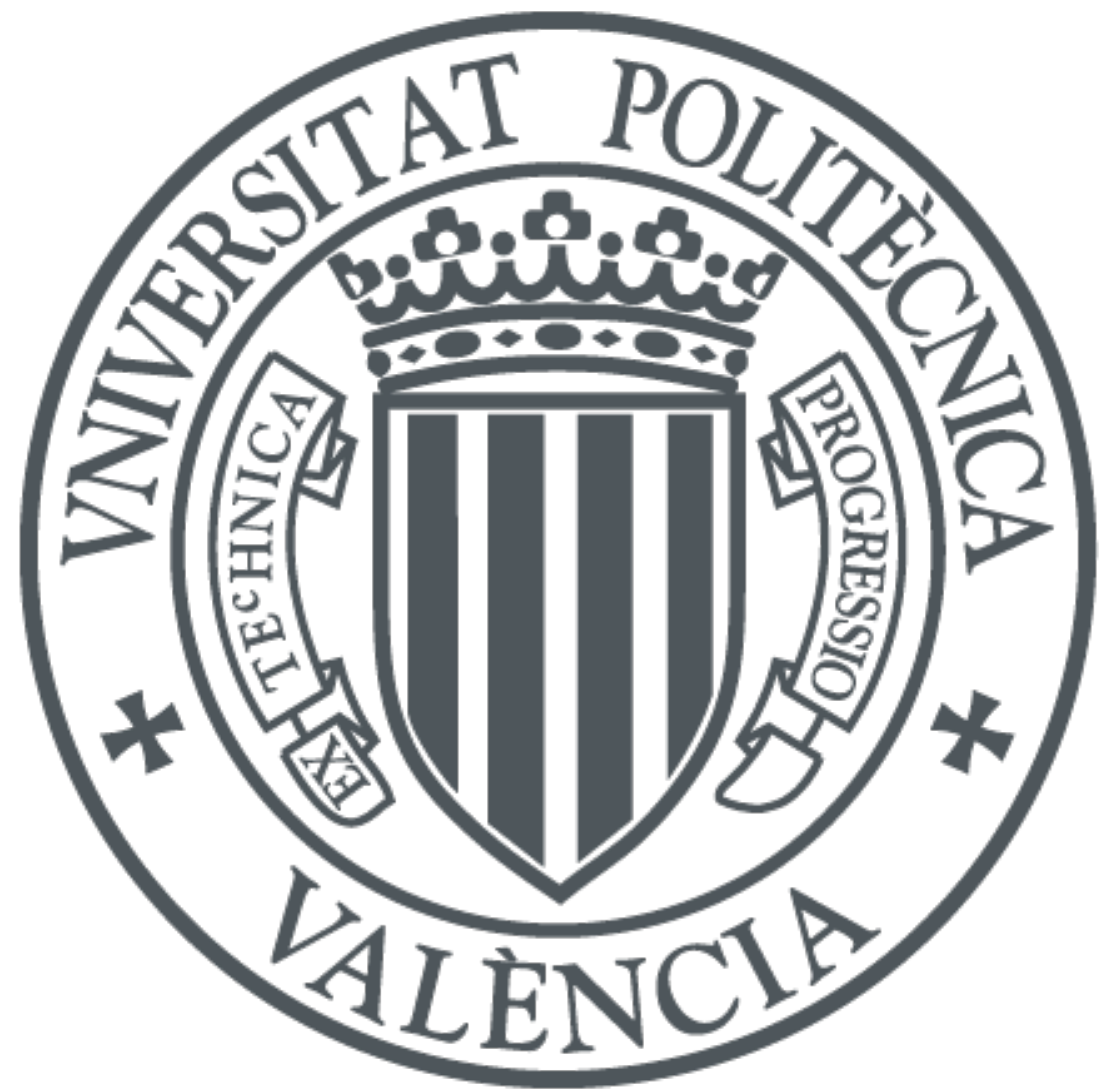

The final publication is available at

https://doi.org/10.1016/j.snb.2018.09.026

Copyright Elsevier

Additional Information 


\title{
Design of Oligonucleotide-capped Mesoporous Silica Nanoparticles for the detection of miRNA-145 by Duplex and Triplex formation
}

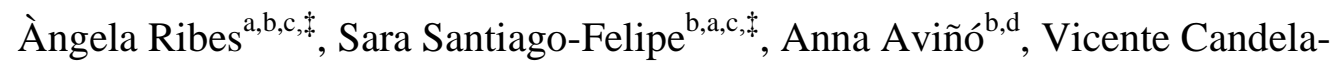
Noguera $^{\text {a,b,c,e }}$, Ramón Eritja ${ }^{\text {b,d* }}$, Félix Sancenón ${ }^{\text {a,b,c,e, }}$, and Ramón Martínez-Máñez ${ }^{\text {a,b,c,e, }}$ and Elena Aznar ${ }^{\mathrm{b}, \mathrm{a}, \mathrm{c}, \mathrm{e}}$

${ }^{a}$ Instituto Interuniversitario de Investigación de Reconocimiento Molecular y Desarrollo Tecnológico, Universitat Politècnica de València, Universitat de València, Camino de Vera s/n, 46022, Valencia, Spain.

${ }^{\mathrm{b}}$ CIBER de Bioingeniería, Biomateriales y Nanomedicina (CIBER-BBN), Spain.

${ }^{\mathrm{c}}$ Unidad Mixta de Investigación en Nanomedicina y Sensores. Universitat Politècnica de València, Instituto de Investigación Sanitaria La Fe, Valencia, Spain

${ }^{\mathrm{d}}$ Institute for Advanced Chemistry of Catalonia (IQAC), CSIC, Jordi Girona 18-26, 08034, Barcelona, Spain.

${ }^{\mathrm{e}}$ Unidad Mixta UPV-CIPF de Investigación en Mecanismos de Enfermedades y Nanomedicina. Universitat Politècnica de València, Centro de Investigación Príncipe Felipe, Valencia, Spain.

$\$$ These authors contributed equally

* Corresponding author:

E-mail: recgma@cid.csic.es; rmaez@qim.upv.es

\begin{abstract}
The development of new strategies to detect microRNAs (miRNAS) has become an important challenge in the biomedical field. We report herein the use of
\end{abstract}


oligonucleotide-gated silica nanoparticles for the detection of miRNA-145. In the proposed design, mesoporous silica nanoparticles (MSNs) are loaded with a fluorescent reporter (rhodamine B) and pores are blocked by specific DNA oligonucleotides. The opening of the gated system and dye delivery is selectively controlled by DNA-miRNA recognition. Moreover, the use of DNA capture probes able to form duplex or triplex structures between target miRNA and the complementary oligonucleotides has been studied. By this simple procedure a limit of detection as low as $0.25 \mathrm{pM}$ was found for miRNA. The method was successfully applied to detect miRNA-145 in serum samples, which demonstrates the high potential of these capped materials to detect miRNA for diagnostic purposes.

\section{Keywords}

Mesoporous silica materials, molecular gates, miRNA-145, biosensor, triplex

\section{Introduction}

MicroRNAs (miRNAs) are a class of small (19-25 nucleotides) non-coding RNA with important roles in gene regulation [1]. These post-transcriptional gene regulators were described for the first time in 1993 and, at this time, a large number of them have been identified. According to the miR database, 28645 miRNA entries in 283 species have been identified so far (http://microrna.sanger.ac.uk/) from which 2661 are unique human miRNAs sequences. miRNAs play an important role in many biological processes including development, cell proliferation, differentiation and apoptosis [1]. Alterative expression of miRNAs has been associated with a number of diseases. Thus, detection of circulating miRNAs present in physiological fluids can provide valuable diagnostic and prognostic data [2]. However, measuring miRNAs is a very challenging task due to their intrinsic properties such as short length, high homologous sequences, low abundance and large variation in base composition and secondary structures [3]. miRNAs are often identified by Northern blotting, microarrays and RT-qPCR, however the practical application of these methods are limited due to their time-consuming and costly labeling techniques, and the expensive and sophisticated instrumental requirements $[4,5]$. In the recent years, and in addition to these traditional approaches, a 
large number of protocols involving non-canonical DNA structures and novel nanomaterials have been deployed [3].

On that regard, the development of efficient and reliable detection strategies for rapid, sensitive and selective identification of miRNAs is of importance. Current works have shown that biosensors technology can offer rapid results with high sensitivity and minimal sample preparations. In this scenario some studies have been published recently based on miRNAs detection by electrochemical or optical biosensors [4, 6-8]. Specifically, those based on the hybridization of target miRNA with a DNA probe used as recognition element, have provided excellent characteristics for clinical diagnosis [9]. All these described methods rely on duplex formats between the miRNA and the complementary DNA probe. Nevertheless, other formats such as triple-helix formation based on Hoogsteen base pairing to the Watson-Crick duplex are also interesting alternatives.

Triplex can only be formed at specific polypurine-polypyrimidine sequences, which are widespread within the human genome, especially at promoter regions $[10,11]$. Previous works have reported the triplex-stabilizing properties of 8-aminopurines and the use of parallel and antiparallel tail-clamps to increase the efficiency of triplex formation with structured DNA and RNA targets $[12,13]$. This triplex affinity capture strategy has been successfully applied for the detection of Listeria mRNA and miRNA-145 using a SPR biosensor $[14,15]$.

From a different point of view, in the last years nanomaterials for sensing applications have played a great role due to their capacity to provide rapid, simple and sensitive responses to target analytes. Among nanomaterials used in sensing protocols, mesoporous silica nanoparticles (MSNs) have been widely employed due to their remarkable properties such as large load capacity, biocompatibility, high inner surface area and the possibility to easily modify their surface. MSNs can be functionalized with a wide range of different (bio)molecules than act as "molecular gates" to obtain hybrid organic-inorganic supports for the design of stimuli-responsive systems [16]. In particular, this type of materials can be used as probes when the support is loaded with a dye and the capping mechanism is designed in a way that only a target analyte is able to trigger the delivery of the cargo [17]. Among the different biomolecules that could act 
as caps, nucleic acids (DNA or aptamers) have shown excellent performances in sensing applications [18-20]. However, as far as we know, the use of DNA-capped silica materials for the detection of miRNAs has not yet been reported.

Based on these concepts, we report herein a new strategy to detect miRNA-145. miRNA-145 is related with several cardiovascular diseases, atherosclerosis and hypertension, and also acts as a tumor suppressor, showing a clear relationship between its expression and clinic pathological findings in human colorectal tumors [21, 22]. Several sensing systems based in gated MSNs loaded with a fluorophore (rhodamine B) and capped with different oligonucleotide probes able to form duplex or triplex structures with miRNA-145 are reported. Best results were found when the capping oligonucleotide used was designed to form an antiparallel triplex, reaching a limit of detection in the low picomolar range. In addition, this direct detection method is compatible with the presence of serum in the samples.

\section{Materials and methods}

\subsection{Synthesis of oligonucleotides}

Oligonucleotides used in this study are listed in Table 1 and they were prepared using phosphoramidite solid phase synthesis protocols as described peviously [12-15]. Briefly, oligonucleotides were asembled on controlled pore glass (CPG) supports by successive addition of the appropriate phosphoramidites using an autmomated Applied Biosystems 394 DNA synthesizer (Foster City, CA, USA). The addition of 8-amino-2'deoxyguanosine was done using the corresponding 8-amino-2'-deoxyguanosine phosphoramidite protected with two dimethylformamidino groups obtained from commercial sources (Berry Associates, USA). The parallel clamps were assembled using standard $3^{6}$-phosphoramidites for the polypurine stretch and reversed $\mathrm{C}$ and $\mathrm{T} 5^{6}$ phosphoramidites and 5'-reversed-C solid support for the C,T-Hoogsteen strand. After the assembly of the sequences, the supports were treated with concentrated ammonia (overnight, $55^{\circ} \mathrm{C}$ ) except for the sequences carrying 8-amino-dG that were treated with $0.1 \mathrm{M}$ mercaptoethanol in concentrated ammonia $\left(24 \mathrm{hr}, 55^{\circ} \mathrm{C}\right)$. RNA oligonucleotides were prepared by standard solid phase protocols using the tert-butyldimethylsilyl group 
for the protection of the $2^{6}-\mathrm{OH}$ group. Oligonucleotides were purified by Glen-PackTM DNA/RNA purification cartridges (Glen Research, USA) and analyzed by mass spectrometry (Table S2, Electronic Supplementary Material).

Table 1. Sequences of the oligonucleotides prepared in this work.

\begin{tabular}{|c|c|c|}
\hline Code & Name & Sequence $\left(5^{\prime}-3^{\circ}\right)$ \\
\hline $\mathbf{0 1}$ & Compl duplex & 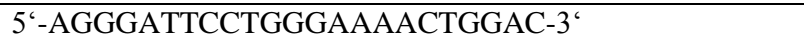 \\
\hline $\mathbf{O 2}$ & Antiparallel-8AG Clamp & 5-AG*G*G*ATTCCTGGGAAAA-TTTT-AAAAGGG-3 ‘ \\
\hline $\mathbf{O 3}$ & Antiparallel Clamp & 5‘-AGGGATTCCTGGGAAAA-TTTT-AAAAGGG-3‘ \\
\hline $\mathbf{O 4}$ & Parallel-8AG Clamp & $5^{\circ}-\mathrm{AG}^{*} \mathrm{G}^{*} \mathrm{G}^{*}$ ATTCCTGGGAAAA-TTTT-3`-3`TTTTCCC-5` \\
\hline $\mathbf{O 5}$ & Parallel Clamp & 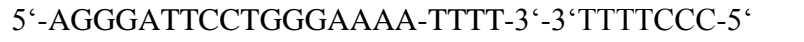 \\
\hline O6 & miRNA-145 & $5^{\prime}-$ GUCCAGUUUUCCCAGGAAUCCCU-3' \\
\hline $\mathbf{O 7}$ & miRNA-141 & 5'-UAACACUGUCUGGUAAAGAUGG-3‘ \\
\hline 08 & miRNA-let 7A & 5'-UGAGGUAGUAGGUUGUAUAGUU-3' \\
\hline
\end{tabular}

\subsection{Synthesis mesoporous silica nanoparticles (MSNs)}

For the synthesis of mesoporous silica nanoparticles ncetyltrimethylammoniumbromide $(\mathrm{CTABr}, 1.00 \mathrm{~g}, 2.74 \mathrm{mmol})$ was first dissolved in $480 \mathrm{~mL}$ of deionized water. Then, $3.5 \mathrm{~mL}$ of $\mathrm{NaOH} 2.00 \mathrm{M}$ in deionized water was added to the CTABr solution, followed by adjusting the solution temperature to $80^{\circ} \mathrm{C}$. TEOS (5 mL, $25.7 \mathrm{mmol}$ ) was then added dropwise to the surfactant solution. The mixture was allowed to stir for $2 \mathrm{~h}$ to give a white precipitate. Finally the solid product was centrifuged, washed with deionized water and dried at $60^{\circ} \mathrm{C}$ (as-synthesized). To prepare the final porous material, the as-synthesized solid was calcined at $550{ }^{\circ} \mathrm{C}$ using oxidant atmosphere for $5 \mathrm{~h}$ in order to remove the template phase.

\subsection{Synthesis of S1}

In a typical synthesis, calcined MSNs (200 mg) and rhodamine B (766.4 mg, 0.16 mmol) were suspended in $\mathrm{CH}_{3} \mathrm{CN}(10 \mathrm{~mL})$ and stirred at room temperature for $24 \mathrm{~h}$. Then (3-aminopropyl)triethoxysilane $(292.5 \mu \mathrm{L}, 1 \mathrm{mmol})$ was added, and the final mixture was stirred at room temperature for $5.5 \mathrm{~h}$. The resulting pink solid (S1) was isolated by filtration, washed with $\mathrm{CH}_{3} \mathrm{CN}(5 \mathrm{~mL})$ and dried at $38{ }^{\circ} \mathrm{C}$ for $18 \mathrm{~h}$. 


\subsection{Synthesis of solids (S2-S6)}

For the preparation of the sensing materials, $500 \mu \mathrm{g}$ of solid $\mathbf{S 1}$ were capped by adding an aqueous solution $(100 \mu \mathrm{M})$ of the corresponding oligonucleotide (O1-O5). Each gated material was optimized to obtain the best performance. Amounts of capping oligonucleotide used to prepare the final materials S2-S6 are shown in Table 2. Volume was completed to $500 \mu \mathrm{L}$ with hybridization buffer $(20 \mathrm{mM}$ Tris- $\mathrm{HCl}, 37.5 \mathrm{mM} \mathrm{MgCl} 2$, $\mathrm{pH} 7.5$ ) and the mixtures were stirred 60 minutes at $37^{\circ} \mathrm{C}$. The resulting materials were isolated by centrifugation, washed with hybridization buffer to eliminate residual dye and free oligonucleotides and dried at vacuum.

Table 2. Volume of the oligonucleotides used to synthesize S2-S6.

\begin{tabular}{llllll}
\hline Solid & S2 & S3 & S4 & S5 & S6 \\
\hline Oligonucleotide & O1 & O2 & O3 & O4 & O5 \\
Volume $(\mu \mathrm{L})$ & 100 & 20 & 10 & 25 & 25 \\
\hline
\end{tabular}

\section{RESULTS AND DISCUSSION}

\subsection{Preparation and characterization of the sensing supports}

MSNs with a diameter of ca. $100 \mathrm{~nm}$ were selected as inorganic scaffolds and pores were loaded with a fluorophore (rhodamine B). This type of reporter has been extensively used in gated materials for the detection of several types of analytes including proteins, small molecules and genomic sequences [17-20]. The external surface was functionalized with (3-aminopropyl)trimethoxysilane, to yield S1. Aminopropyl moieties are partially charged at a neutral $\mathrm{pH}$ and are known to display electrostatic interactions with negatively charged oligonucleotides [18, 19]. Based on this, addition of oligonucleotides O1-O5 to solution of S1 yielded the capped solids S2S6. It was expected that in the presence of miRNA145, the capping oligonucleotide will be displaced to form a hibridized structure with miRNA145, allowing the release of the entrapped dye as depicted in Scheme 1. 


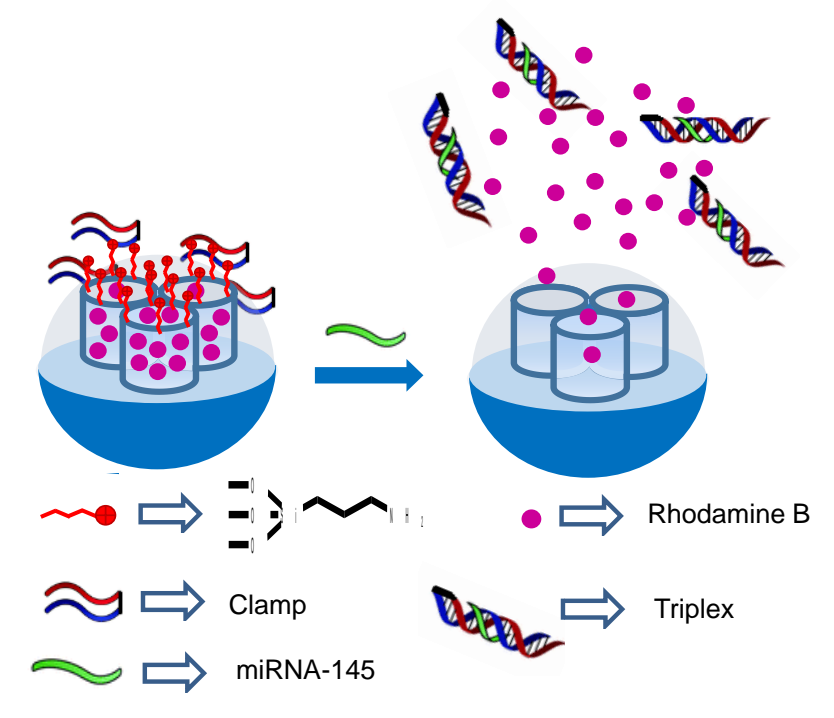

Scheme 1. Representation of the gated materials capped with oligonucleotides. Delivery of the entrapped rhodamine B was selectively observed in the presence of miRNA-145 via formation of duplex or triplex structures.

The starting MSNs and the synthetized solids S1-S6 were characterized following standard techniques, including powder X-ray diffraction (PXRD), transmission electron microscopy (TEM), $\mathrm{N}_{2}$ adsorption-desorption isotherms, thermogravimetric analysis (TGA), and dynamic light scattering (DLS).

PXRD and TEM carried out on calcined MSNs clearly showed the presence of a mesoporous structure that persisted in solids S1-S6 regardless of the loading of the porous with rhodamine $\mathrm{B}$, further functionalization with (3aminopropyl)trimethoxysilane and capping with the oligonucleotides 01-05 (see Figure S-1 in the ESM). BET specific surface area, pore volume and pore size were calculated from $\mathrm{N}_{2}$ adsorption-desorption measurements [23, 24]. For the starting calcined MSNs a value of BET specific surface of $1027.53 \mathrm{~m}^{2} \mathrm{~g}^{-1}$ was obtained, while for $\mathbf{S 1}$ a decrease of the specific surface was observed $\left(278.21 \mathrm{~m}^{2} \mathrm{~g}^{-1}\right)$, which is agreement with the presence of the rhodamine B dye inside the pores and surface functionalization (see Figure S-2 and Table S-1 in the ESM). Size of nanoparticles was determined by dynamic light scattering (DLS) studies. Diameters of 105.7, 141.8, 278.6, 275.9, 289.8, 296.9 and $295 \mathrm{~nm}$ were found for starting calcined MSNs, S1, S2, S3, S4, S5 and S6, respectively, showing a hydrodynamic diameter increase from calcined MSNs to S1, and from S1 to final sensing solids S2-S6 (see Figure S-3 in the 
ESM). Moreover, the content of 3-aminopropyl moieties, rhodamine B, 01, 02, O3, $\mathbf{O 4}$ and $\mathbf{O 5}$ in the different prepared solids was calculated from thermogravimetric analysis (Table 3).

Table 3. Contents $\left(\mathrm{mmol} \mathrm{mg}^{-1}\right)$ of 3-aminopropyl, rhodamine B, O1, O2, O3, $\mathbf{O 4}$ and $\mathbf{O 5}$ in the different prepared solids.

\begin{tabular}{llllllll}
\hline & Rhodamine B & 3-aminopropyl & O1 & O2 & O3 & O4 & O5 \\
\hline S1 & $6.86 \cdot 10^{-3}$ & $5.90 \cdot 10^{-2}$ & - & - & - & - & - \\
S2 & $2.90 \cdot 10^{-3}$ & $5.70 \cdot 10^{-2}$ & $2.50 \cdot 10^{-4}$ & - & - & - & - \\
S3 & $2.57 \cdot 10^{-3}$ & $5.70 \cdot 10^{-2}$ & - & $2.20 \cdot 10^{-4}$ & - & - & - \\
S4 & $2.47 \cdot 10^{-6}$ & $5.80 \cdot 10^{-2}$ & - & - & $2.05 \cdot 10^{-4}$ & - & - \\
S5 & $2.43 \cdot 10^{-6}$ & $5.90 \cdot 10^{-2}$ & - & - & - & $1.93 \cdot 10^{-4}$ & - \\
S6 & $1.83 \cdot 10^{-6}$ & $6.00 \cdot 10^{-2}$ & - & - & - & - & $2.58 \cdot 10^{-4}$ \\
\hline
\end{tabular}

\subsection{Synthesis of oligonucleotide capture probes for duplex and triplex formation}

Based on our previous work on triplex-affinity capture of miRNA-145 for SPR detection [15], several DNA probes carrying a complementary sequence to the polypyrimidine track (5'-UUUUCCC-3') of miRNA-145 were prepared (Table 1, Scheme 2). The complementary sequence (O1, duplex) is a 23-bases long oligonucleotide designed to form the full 23-bases long duplex (Scheme 2). The antiparallel clamps $(\mathbf{O 2}, \mathbf{O 3})$ contain a complementary sequence of 17 bases followed by a tetrathymidine loop and the reverse Hoogsteen sequence (5'-AAAAGGG-3', Scheme 2).
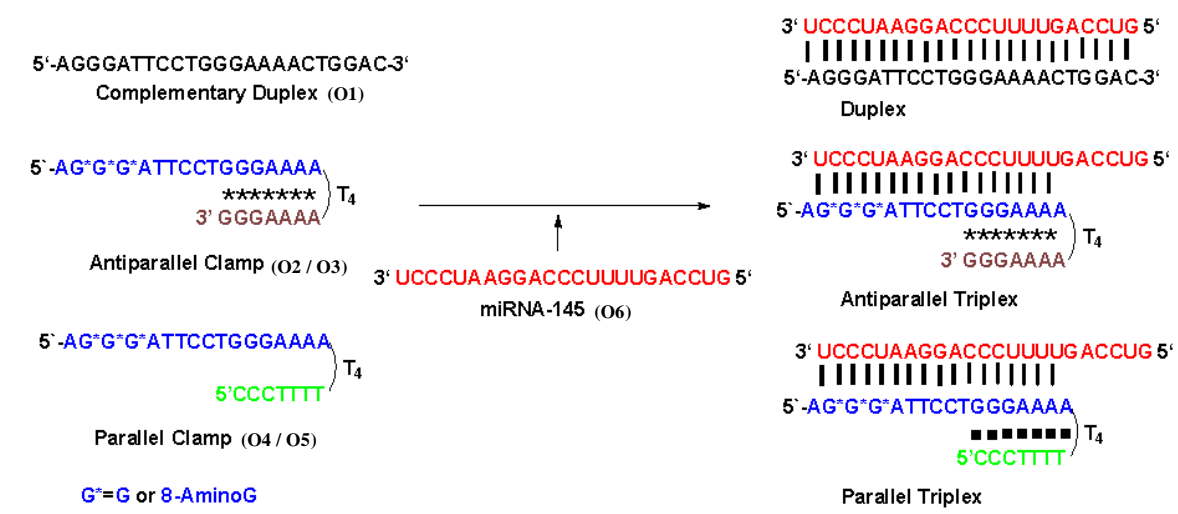

Scheme 2. Schematic representation of binding of miRNA-145 with complementary duplex and Antiparallel and Parallel Clamps to form duplex and Antiparallel and Parallel triplex. 
Oligonucleotide $\mathbf{O 2}$ carries three modified 8-aminoguanine residues replacing 3 guanines (see Table 1). It has been described that the change of guanine by 8 aminoguanine may increase the stability of the antiparallel triplex as shown in thermal denaturation studies [23]. These oligonucleotides $(\mathbf{O 2}, \mathbf{O 3})$ are designed to form an antiparallel triplex with miRNA-145. The parallel clamps $(\mathbf{O 4}, \mathbf{O 5})$ contain the same complementary sequence of 17 bases followed by a tetrathymidine loop and the Hoogsteen sequence (3'-TTTTCCC-5'). The Hoogsteen sequence has the opposite polarity to the Watson-Crick sequence and has been characterized by denaturation studies [15]. Thus, these oligonucleotides $(\mathbf{O 4}, \mathbf{O 5})$ are able to form a parallel triplex with miRNA-145. Oligonucleotide clamp $\mathbf{O 4}$ carries also the three modified 8aminoguanine residues because it has been described that parallel triplexes are stabilized by the 8-amino group of the guanine [12]. All together, these DNA capture probes will show whether the formation of parallel or antiparallel triplex is advantageous or not in the capture of the target microRNA and subsequent release of the dye.

We also expect to answer whether the stabilization properties of triplex of 8aminopurines observed in solution is maintained or not in the capture of the target miRNA on the surface of mesoporous materials.

\subsection{Release experiments}

In a first step, the performance of solids $\mathbf{S 2}, \mathbf{S 3}, \mathbf{S 4}, \mathbf{S 5}$ and $\mathbf{S 6}$ was studied. A $500 \mu \mathrm{g}$ fraction of each solid was suspended with hybridization buffer and separated in 2 aliquots. Then, $10 \mu \mathrm{L}$ an aqueous solution of miRNA-145 $(100 \mu \mathrm{M})$ were then added to one of the aliquots whereas $10 \mu \mathrm{L}$ of water were added to the other aliquot. Suspensions were maintained under stirring and at fixed times (i.e. 10, 20, 35, 50, $60 \mathrm{~min}$ ) fractions were taken and centrifuged to separate the solid from the aqueous phase. Cargo release was then determined by measuring emission of rhodamine $\mathrm{B}$ dye delivered from the pores to the solution. As an example, Figure 1 shows the delivery kinetics profile of rhodamine B from solid S4 in the presence and in the absence of miRNA-145. In the absence of miRNA-145 (curve a), a poor rhodamine B release (less than $10 \%$ in one 
hour) was observed, indicating tight pore closure. In contrast, when miRNA-145 was present in the solution, the delivery of the dye was induced due to the displacement of the capping oligonucleotide from the nanoparticle to the solution (curve b).

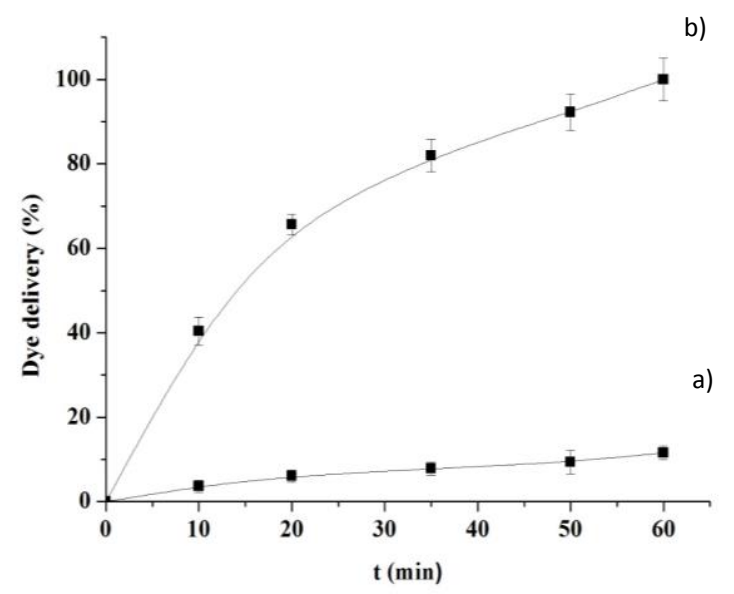

Figure 1. Release profile of rhodamine B from solid S4 in the absence (a) and the presence (b) of miRNA-145.

A similar response was observed for solids $\mathbf{S 2}, \mathbf{S 3}, \mathbf{S 5}$ and $\mathbf{S 6}$, showing a selective pore opening and dye delivery regardless the recognition between the oligonucleotide and miRNA-145 formed a duplex or a triplex structure (see Figure S-4 in the ESM). As depicted in Figure S-4, dye delivery from solids capped with oligonucleotides designed to form an antiparallel triplex (S3 and S4), showed better performance and a slightly faster response to the presence of miRNA-145. In addition, in the case of solids $\mathbf{S 3}$ and S4, a flat release curve was observed in the absence of miRNA-145 (curves a), indicating a more stable pore closure. These differences are an indication of the efficiency of triplex or duplex formation, as the release of the dye is caused by the displacement of the capped oligonucleotide from the surface due to triplex or duplex formation. Antiparallel triplexes shaped when using solids $\mathbf{S 3}$ and $\mathbf{S 4}$, can be more rapidly formed than duplexes and parallel triplexes. Also, it is important to notice than duplex probe in $\mathbf{S} 2$ is 23 base pairs long while the triplex probes are only 17 base pairs long. Despite that, antiparallel triplexes are formed more efficiently than duplex and parallel triplex. The more efficient release of the antiparallel triplexes (solids $\mathbf{S 3}$ and $\mathbf{S 4}$ ) may be due to the fact that antiparallel tail-clamps have the reversed Hoogsteen duplex 
structure already formed before the addition of the target RNA. This may facilitate the capture of miRNA oligonucleotide to form the corresponding antiparallel triplex. The formation of parallel triplexes needs the protonation of cytosines that occur at acid $\mathrm{pH}$ [24]. We have analyzed the release of parallel triplex (solids S5 and S6) at pH 5.5 but we were unable to observe any improvement at this $\mathrm{pH}$. On the other hand, no significant differences are observed with the presence of the 8-aminoguanine residue, indicating that the higher stability of the triplex observed in thermal denaturation studies $[12,25]$ does not increase the efficiency of dye release in the present system.

\subsection{Analytical performance: sensitivity and specificity studies}

To assess the sensitivity of the method, the response of S2-S6 to different concentrations of miRNA-145 was studied. Thus, suspensions of solids were treated with different amounts of a miRNA-145 standard solution. It was found that the amount of delivered rhodamine B was proportional to miRNA-145 concentration in all cases, which is in agreement with the uncapping protocol detailed above (see Figure S-5 in the ESM). Results of the sensitivity obtained with all the sensing solids are displayed in Table 4. A limit of detection of $2.5 \mathrm{pM}, 5.0 \mathrm{pM}$ and $8.0 \mathrm{pM}$ was calculated for duplex (solid S2), parallel triplex (solid S5) and parallel-8Ag triplex (solid S6), respectively, whereas antiparallel (solid S3) and antiparallel-8Ag triplex (solid S4) exhibited more than 10 times lower LODs $(0.30 \mathrm{pM}$ and $0.25 \mathrm{pM}$, respectively). The higher sensitivity of the solids carrying the antiparallel probes (S3 and $\mathbf{S 4})$ is in agreement with the release experiments described above. The higher sensitivity provided by the antiparalel triplex format (solids $\mathbf{S 3}$ and $\mathbf{S 4}$ ) may be due to a combined effect of the structure of the antiparallel clamps, which are more efficient in bloking MSNs, and a more rapid formation of the triplex due to the existence of a preformed reversed Hoogsteen antiparalel structure in the capping oligonucleotides $\mathbf{O 2}$ and 03. The obtained LODs fell within the expected concentrations for circulating miRNAs in human serum (0-1 pM), demonstrating the potential of the method for its application in clinical diagnostics. Compared with the SPR protocol using similar triplex-forming capture probes [15] the systems described herein show an increase of 3 orders of magnitude. This increase could be ascribed to the amplification of the signal induced by the release of a large number of fluorescent rhodamine molecules per recognition event when using gated 
materials. The limits of detection obtained are in the range of Northern blotting or microarray [2], however, our proposed method is simpler, faster and no especial instrumentation is needed nor labeling of the sample.

Table 4. Limits of detection of solids S2-S6.

\begin{tabular}{llc}
\hline Solid & Oligonucleotide & Limit of Detection $(\mathrm{pM})$ \\
\hline S2 & Duplex & 2.5 \\
S3 & Antiparallel-8Ag & 0.30 \\
S4 & Antiparallel & 0.25 \\
S5 & Parallel-8Ag & 5.0 \\
S6 & Parallel & 8.0 \\
\hline
\end{tabular}

In a step forward, the specificity of the solids was confirmed by studying the response of S2-S6 to different possible interferent miRNAs such as miRNA-Let7A and miRNA141. Thus, the response of the different solids was studied in the presence of each miRNA $(0.1 \mu \mathrm{M})$. As it is shown in Figure 2, miRNA-145 is the only molecule able to induce a notable dye delivery, whereas all the interferents induced less pore opening and poor payload release. This observation supports a selective oligonucleotide-miRNA-145 interaction as mechanism of the observed optical response, and corroborates the preferential binding of the used oligonucleotides with the miRNA-145 sequence, which resulted in uncapping of the pores and payload delivery.

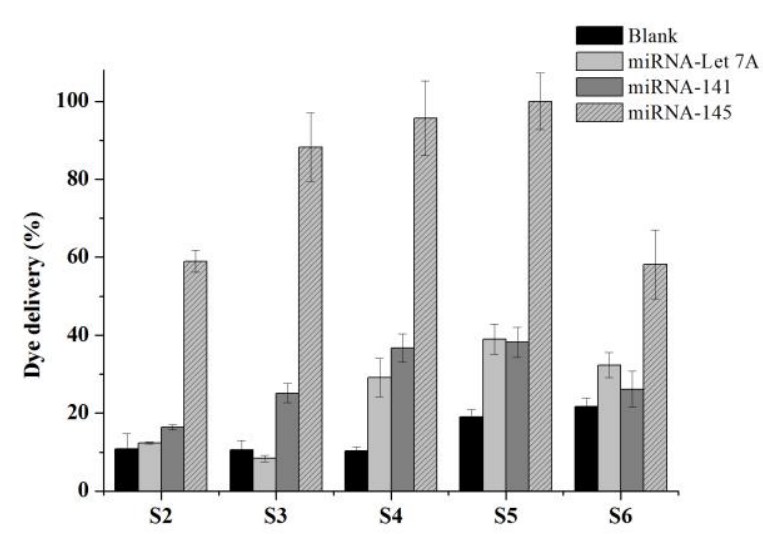

Figure 2. Release of rhodamine B from solid S2, S3, S4, S5 and S6 in the absence and the presence of miRNA-Let7A, miRNA-141 and miRNA-145.

\section{5 miRNA-145 detection in real media}


The detection of miRNA-145 directly in real samples is a topic of high interest. New strategies to help medical professionals to obtain accurate and rapid results to early diagnose and made a prognosis of their patients is a decidedly pursued goal. Therefore, the potential use of the sensing solids to detect miRNA-145 in more competitive samples was studied. In particular, delivery of rhodamine B from S2 and S4 in the presence and in the absence of miRNA-145 was carried out in human serum samples (see Experimental section for details). Figure 3 shows a selective displacement of the oligonucleotide, pore opening and dye release in the presence of miRNA-145 for both solids after $30 \mathrm{~min}$ (grey columns). Although a slightly higher background was observed in serum samples when compared with that obtained in hybridization buffer (ca. 20\% and 10\%, respectively), a clear larger amount of dye was delivered in the presence of miRNA-145 which resulted in accurate miRNA-145 detection. These results show that the proposed method may be a promising alternative to classical procedures for the detection of target miRNA in clinical relevant samples.

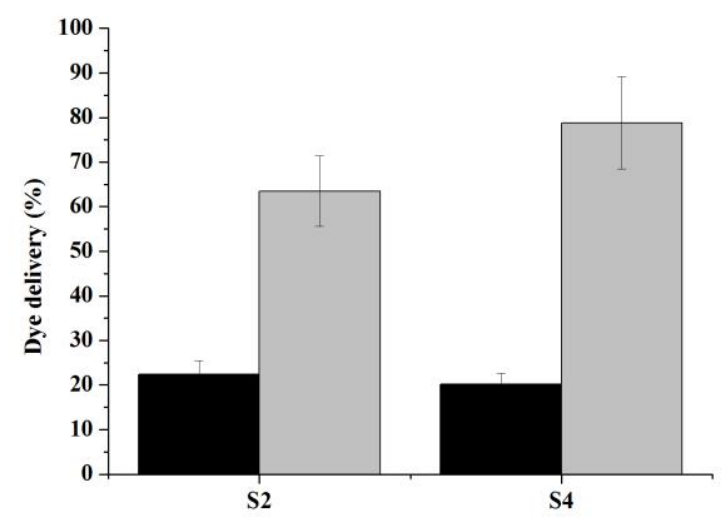

Figure 3. Released rhodamine B from solid S2 and S4 in the absence (in black) and the presence (in grey) of miRNA-145 in human serum samples.

\section{Conclusions}

Development of new strategies for sensitive and label-free detection of miRNA has become an important issue in the clinical field. Here we present a new protocol for the detection of miRNA-145 by using MSNs loaded with a dye and capped with specific 
oligonucleotides. The response of the detection systems is related with the recognition between the capping oligonucleotide and miRNA-145, which induced a displacement of the oligonucleotide from the solid, pore opening and dye release. The use of duplex or triple-helix formats between the miRNA and the complementary DNA probe was studied. In particular, the use of antiparallel and parallel clamps and the effect of introducing 8-amino-2'-deoxyguanosine in these clamps were analyzed. All proposed solids presented excellent analytical performances in terms of sensitivity and selectivity. Specifically, the antiparallel triplex formation (S3 and $\mathbf{S 4}$ ) resulted in a more efficient approach when compared with parallel triplex or duplex formation, reaching LODs as low as $0.25 \mathrm{pM}$. In addition, the capped nanomaterial allowed an accurate determination of miRNA-145 in realistic human serum samples. The achieved results are of interest towards the detection of miRNA in a realistic environment. The method is simple, portable, can be easily tuned via the use of different reporting molecules (e.g. dyes, fluorophores or electroactive species) and may allow the development of simple tests, offering a great potential for clinical applications.

\section{Acknowledgements}

We thank the Spanish Government (projects MAT2015-64139-C4-1-R and CTQ201452588-R (MINECO/FEDER)), the Generalitat Valenciana (project PROMETEOII/2014/047) and the Generalitat de Catalunya (2014/SGR/624) for support. A.R. thanks UPV for her predoctoral fellowship. S.S. thanks the Instituto de Salud Carlos III and the European Social Fund for the financial support "Sara Borrell" (CD16/000237). V.C.-N. thanks Ministerio de Educación, Cultura y Deporte for his predoctoral grant. The authors also thank the Electron Microscopy Service at the UPV for support.

Electronic Supplementary Material: Supplementary material about experimental and characterization details, release experiments and limit of detection is available in the online version of this article at http://dx.doi.org/10.1007/s12274_***_****_* (automatically inserted by the publisher). 


\section{References}

[1] (a) S.L. Ameres, P.D. Zamore, Diversifying microRNA sequence and function. Nat. Rev. Mol. Cell Biol., 14 (2013) 475-488. (b) S. Jonas, E. Izaurralde, Towards a molecular understanding of micro-RNA-mediated gene silencing. Nat Rev. Genetics, 16 (2015) 421-433. (c) A. Alagia, E. Eritja, The biochemistry of gene silecing. Encyclopedia Life Sciences, eLS, John Willey \& Sons, Ltd. Chichester, 2017, a0021019.

[2] H. Dong, J. Lei, L. Ding, Y. Wen, H. Ju, X. Zhang, MicroRNA: function, detection, and bioanalysis. Chem. Rev., 113 (2013) 6207-6233.

[3] (a) T. Tian, J. Wang, X. Zhou, A review: microRNA detection methods. Org. Biomol. Chem., 13 (2015) 2226-2238. (b) M. Mahdiannasser, Z. Kamari, An innovative paradigm of methods in microRNAs detection; highlighting DNAzymes, the illuminators. Biosensors Bioelect., 107 (2018) 123-144.

[4] L. Liu, N. Xia, H. Liu, X. Kang, X. Liu, C. Xue, X. He, Highly sensitive and labelfree electrochemical detection of microRNAs based on triple signal amplification of multifunctional gold nanoparticles, enzymes and redox-cycling reaction. Biosens. Bioelect., 53 (2014) 399-405.

[5] M. Kaplan, T. Kilic, G. Guler, J. Mandli, A. Amine, M. Ozsoz, A novel method for sensitive microRNA detection: Electropolymerization based doping, Biosens. Bioelect., 92 (2017) 770-778.

[6] F. Khakbaz, M. Mohamad, Micro-RNA detection based on fluorescence resonance energy transfer of DNA-carbon quantum dots probes, Anal. Biochem., 523 (2017) 3238.

[7] S. Azzouzi, W.C. Mak, K. Kor, A.P.F. Turner, M.B. Ali, V. Beni, An integrated dual functional recognition/amplification bio-label for the one-step impedimetric detection of Micro-RNA-21, Biosens. Bioelect., 92 (2017) 154-161.

[8] T. Kilic, A. Erdem, M. Ozsoz, S. Carrara, microRNA biosensors: Opportunities and challenges among conventional and commercially available techniques, Biosens. Bioelect., 99 (2018) 525-546. 
[9] S. Fang, H.J. Lee, A.W. Wark, R.M. Corn, Attomole microarray detection of microRNAs by nanoparticle-amplified SPR imaging measurements of surface polyadenylation reactions, J. Am. Chem. Soc., 128 (2006) 14044.

[10] J.R. Goñi, X. De La Cruz, X. Orozco, Triplex forming oligonucleotide target sequences in the human genome, Nucleic acids res., 32 (2004) 354-360.

[11] J.R. Goñi, J.M. Vaquerizas, J. Dopazo, M. Orozco, Exploring the reasons for the large density of triplex-forming oligonucleotide target sequences in the human regulatory regions, BMC genomics, 7 (2006) 63.

[12] A. Aviñó, M. Frieden, J.C. Morales, B. García de la Torre, R. Güimil Garcia, F. Azorín, J.L. Gelpi, M. Orozco, C. González, R. Eritja, Properties of triple helices formed by parallel-stranded hairpins containing 8-aminopurines, Nucleic Acids Res., 30 (2002) 2609-2619.

[13] A. Nadal, R. Eritja, T. Esteve, M. Pla, "Parallel” and "Antiparallel Tail-Clamps. Increase the efficiency of triplex formation with structured DNA and RNA targets. ChemBioChem., 6 (2005) 1034-1042.

[14] L.G. Carrascosa, S. Gomez-Montes, A. Aviño, A. Nadal, M. Pla, R. Eritja, L.M. Lechuga, Sensitive and label-free biosensing of RNA with predicted secondary structures by a triplex affinity capture method, Nucleic Acids Res., 40 (2012) 56.

[15] A. Aviñó, C.S Huerta, L.M. Lechuga, R. Eritja, Sensitive and label-free detection of miRNA-145 by triplex formation, Anal. Bioanal. Chem., 408 (2016) 885-893.

[16] (a) E. Aznar, M. Oroval, L. Pascual, J.R. Murguía, R. Martinez-Máñez, F. Sancenón, Gated materials for on-command release of guest molecules, Chem. Rev., 116 (2016) 561-718 (b) J. Wen, K. Yang, F. Liu, H. Li, J. Xu, S. Sun, Diverse gatekeepers for mesoporous silica nanoparticle based drug delivery systems, Chem. Soc. Rev., 46 (2017) 6024. (c) C. Li, M. Qian, S. Wang, H. Jiang, Y. Du, J. Wang, W. Lu, N. Murthy, R. Huang, Aptavalve-gated Mesoporous Carbon Nanospheres image Cellular Mucin and provide On-demand Targeted Drug Delivery. Theranostics, 7 (2017) 3319. (d) R. Bhat, I. García, E. Aznar, B. Arnaiz, M.C. Martínez-Bisbal, L.M. Liz-Marzán, S. Penadés, R. Martínez-Máñez, Lectin-gated and glycan functionalized mesoporous silica nanocontainers for targeting cancer cells overexpressing Lewis $\mathrm{X}$ antigen. Nanoscale., 10 (2018) 239-249. (e) T. Ribeiro, E. Coutinho, A.S. Rodrigues, C. 
Baleizão, J.P.S. Farinha, Hybrid mesoporous silica nanocarriers with thermovalveregulated controlled release. Nanoscale., 9 (2017) 13485.

[17] (a) F. Sancenón, L. Pascual, M. Oroval, E. Aznar, R. Martínez-Máñez, Gated silica mesoporous materials in sensing applications, ChemistryOpen, 4 (2015) 418-437. (b) Q. Tan, R. Zhang, R. Kong, W. Kong, W. Zhao, F. Qu, Detection of glutathione based on $\mathrm{MnO} 2$ nanosheet-gated mesoporous silica nanoparticles and target induced release of glucose measured with a portable glucose meter. Microchim. Acta, 185 (2018) 44. [18] (a) M. Ercan, V.C. Ozalp, B.G. Tuna, Genotyping of single nucleotide polymorphism by probe-gated silica nanoparticles. Anal. Biochem., 537 (2017) 78. (b) L. Pascual, I. Baroja, E. Aznar, F. Sancenón, M.D. Marcos, J.R. Murguia, P. Amoros, K. Rurack, R. Martinez-Máñez, Oligonucleotide-capped mesoporous silica nanoparticles as DNA-responsive dye delivery systems for genomic DNA detection. Chem. Comm., 51 (2015) 1414-1416.

[19] P. Zhang, F. Cheng, R. Zhou, J. Cao, J. Li, C. Burda, Q. Min, J.J. Zhu, DNAhybrid-gated multifunctional mesoporous silica nanocarriers for dual-targeted and microRNA-responsive controlled drug delivery. Angew. Chem. Int. Ed., 53 (2014) 2371-2375.

[20] (a) Z. Zhang, F. Wang, D. Balogh, I. Willner, pH-controlled release of substrates from mesoporous $\mathrm{SiO} 2$ nanoparticles gated by metal ion dependent DNAzymes J. Mater. Chem. B, 2 (2014) 4449. (b) A. Aviñó, M. D. Marcos, F. Sancenón, R. MartínezMáñez, P. Amorós, An aptamer-gated silica mesoporous material for thrombin detection. Chem. Comm., 49 (2013) 5480-5482. (c) A. Ribes. E. Xifré-Pérez, E. Aznar, F. Sancenón, T. Pardo, L.F. Marsal, R. Martínez-Máñez, Molecular gated nanoporous anodic alumina for the detection of cocaine, Sci. Rep., 6 (2016) 38649.

[21] D. Santovito, C. Mandolini, P. Marcantonio, V. de Nardis, M. Bucci, C. Paganelli, Overexpression of microRNA-145 in atherosclerotic plaques from hypertensive patients, Expert Opin. Ther. Targets, 17 (2013) 217-223.

[22] T. Takagi, A. Iio, Y. Nakagawa, T. Naoe, N. Tanigawa, Y. Akao, Decreased expression of microRNA-143 and-145 in human gastric cancers, Oncology, 77 (2009) $12-21$. 
[23] A. Aviñó, E. Cubero, C. González, R. Eritja, M. Orozco, Antiparallel triple helices. Structural characteristics and stabilization by 8-amino derivatives, J. Am.

Chem. Soc., 125 (2003) 16127-16138.

[24] J. Robles, A. Grandas, E. Pedroso, F.J. Luque, R. Eritja, Orozco, M. Nucleic acid triple helices: Stability effects of nucleobase modifications, Curr. Org. Chem., 6 (2002) 1333-1368. 